\title{
Norberto Luiz Cabral, MD, PhD - (1963-2019)
}

\author{
Norberto Luiz Cabral, MD, PhD - (1963-2019) \\ Ylmar CORREA NETO', Hélio Afonso Ghizoni TEIVE²
}

${ }^{1}$ Universidade Federal de Santa Catarina, Hospital Universitário, Departamento de Medicina Interna, Serviço de Neurologia, Florianópolis SC, Brazil.

${ }^{2}$ Universidade Federal do Paraná, Hospital de Clínicas, Departamento de Medicina Interna, Serviço de Neurologia, Curitiba PR, Brazil. Ylmar CORREA NETO (iD https:// orcid.org/0000-0001-7032-5300; Hélio Afonso Ghizoni TEIVE (iD) https://orcid.org/0000-00032305-1073

Correspondence: Hélio A. Ghizoni Teive; Rua General Carneiro, 1103 / 102; 80060150 Curitiba PR, Brazil; E-mail: hagteive@mps.com.br Conflict of interest:

The authors declare no conflict of interest.

Received on December 16, 2019; Accepted on December 23, 2019

\section{(c) BY}

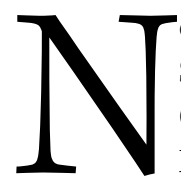
orberto Luiz Cabral was born in 1963 in São Francisco do Sul City, Santa Catarina State, Brazil. He graduated in Medicine from Universidade Federal de Santa Catarina, Florianópolis City, in 1988, where he also did his Internal Medicine Medical Residence during 1989 and 1990. His training in Neurology occurred between 1991 and 1993 at Universidade Federal do Paraná, Neurology Residence Program, Curitiba City, Paraná State. In there, he acquired his Master's Degree in Internal Medicine in 2003, with the dissertation "Study comparing the stroke unit outcome and conventional ward treatment: a randomized study in Joinville, Brazil"'. In 2008, he acquired his PhD Degree, at Universidade de São Paulo, São Paulo City, with the thesis "Evaluation of impact, mortality and lethality for cerebrovascular disease in Joinville, Brazil: comparison between 1995 and the period 2005-6 (JOINVASC Project) ${ }^{\prime 2.3}$. In 2011, he made a post-doctoral fellowship at University of Edinburgh, UK.

Always restless and inquisitor, Cabral, as he was called, published 52 articles and six book chapters on stroke and related problems. Fifteen articles were published by Archives of Neuro Neuropsychiatry $1,4,5,5,7,8,9,10,11,12$, including his publication debut, as many Brazilian researchers, in $1997^{13}$. In 2008 he received from the Brazilian Academy of Neurology the Award of Best Thesis of the Year, and in 2011 he received an International Scholarship by the British Association and the Brazilian Academy of Neurology.

JOINVASC was his most cherished project. Cabral and other neurologists developed a comprehensive project on Stroke Care in Joinville City, Santa Catarina State, including high-quality assistance, research and teaching. He also involved the community and the local government in prevention with

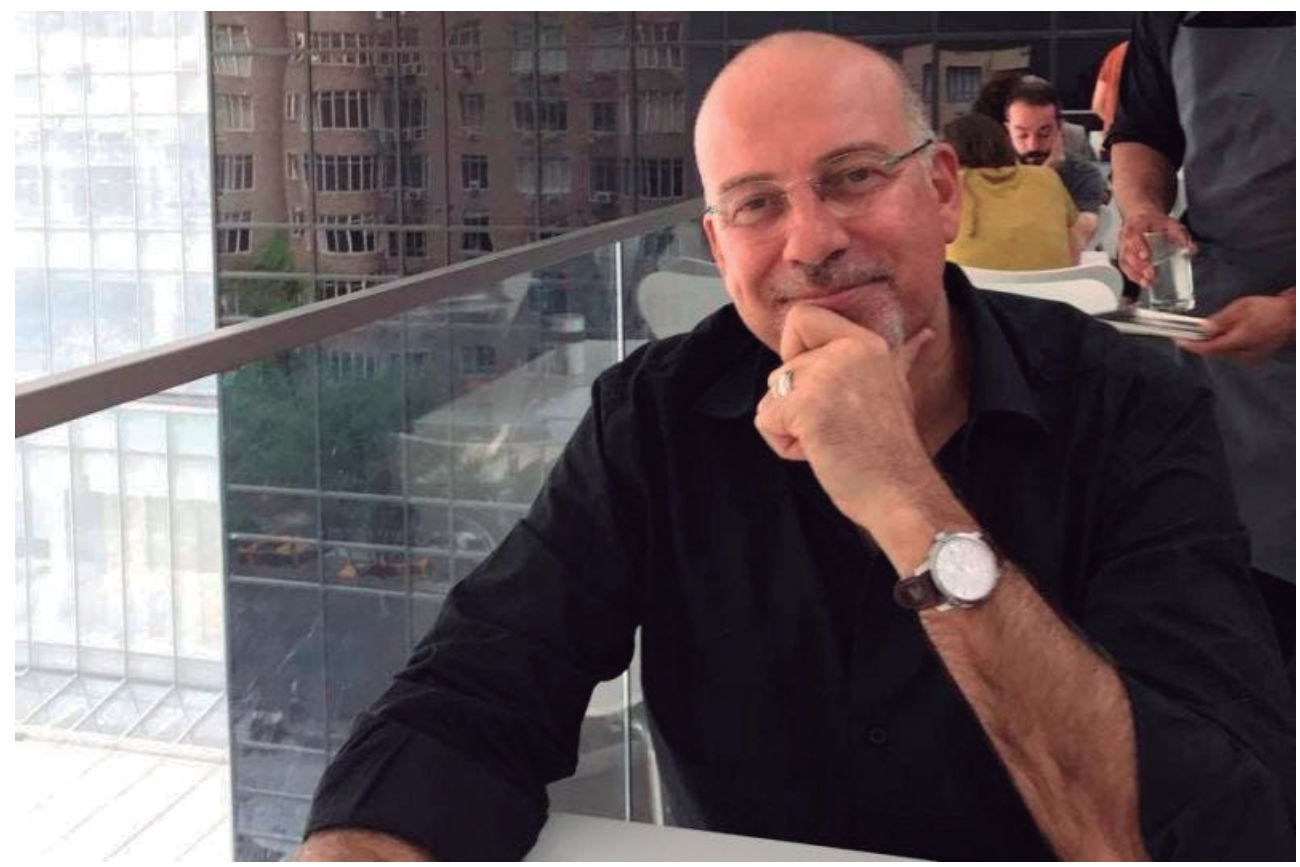

Norberto Luiz Cabral, MD, PhD. 
public education motivational campaigns ${ }^{14,15,16,17,18,19,20,21,22,23,24,25}$. His Stroke Unit was one of the first ones in Brazil.

Cabral was a full professor of Neurology at Universidade de Joinville, acting on graduation and post-graduation programs and becoming an inspiring figure for local students. Cabral died in a car accident in September $27^{\text {th }}, 2019$, aged 56. Cabral will be missed by his family, friends, students, residents, and particularly by his patients.

\section{References}

1. Cabral NL, Moro C, Silva GR, Scola RH, Werneck LC. Study comparing the stroke unit outcome and conventional ward treatment: a randomized study in Joinville, Brazil. Arq Neuropsiquiatr. 2003 Jun;61 (2A):188-93. https://doi.org/10.1590/S0004-282X2003000200006

2. Cabral NL, Gonçalves AR, Longo AL, Moro CH, Costa G, Amaral CH, et al. Incidence of stroke subtypes, prognosis and prevalence of risk factors in Joinville, Brazil: a 2 year community based study. J Neurol Neurosurg Psychiatry. 2009 Jul;80(7):755-61. https://doi.org/10.1136/ jnnp.2009.172098

3. Cabral NL, Gonçalves AR, Longo AL, Moro CH, Costa G, Amaral CH, et al. Trends in stroke incidence, mortality and case fatality rates in Joinville, Brazil: 1995-2006. J Neurol Neurosurg Psychiatry. 2009 Jul;80(7):749-54. https://doi.org/10.1136/jnnp.2008.164475

4. Fonseca PG, Weiss PA, Harger R, Moro CH, Longo AL, Gonçalves AR, et al. Transient ischemic attack incidence in joinville, Brazil, 2010: a population-based study. Stroke. 2012 Apr;43(4):1159-62. https://doi. org/10.1161/STROKEAHA.111.645101

5. Cabral NL, Volpato D, Ogata TR, Ramirez T, Moro C, Gouveia S. Fibrilação atrial crônica, AVC e anticoagulação: sub-uso de warfarina? [Atrial fibrillation, stroke and anticoagulation: under-use of warfarin?]. Arq Neuropsiquiatr. 2004 Dec;62(4):1016-21. https:// doi.org/10.1590/S0004-282X2004000600016

6. Lange MC, Cabral NL, Moro CH, Longo AL, Gonçalves AR, Zétola $V F$, et al. Incidence and mortality of ischemic stroke subtypes in Joinville, Brazil: a population-based study. Arq Neuropsiquiatr. 2015 Aug;73(8):648-54. https://doi.org/10.1590/0004-282X20150081

7. Amaral CHD, Amaral AR, Nagel V, Venancio V, Garcia AC, Magalhães PSC, et al. Incidence and functional outcome of atrial fibrillation and non-atrial fibrillation- related cardioembolic stroke in Joinville, Brazil: a population-based study. Arq Neuropsiquiatr. 2017 May;75(5):288-94. https://doi.org/10.1590/0004-282×20170039

8. Ferreira LE, França PHC, Nagel V, Venancio V, Safanelli J, Reis FID, et al. Joinville stroke biobank: study protocol and first year's results. Arq Neuropsiquiatr. 2017 Dec;75(12):881-9. https://doi. org/10.1590/0004-282X20170157

9. Safanelli J, Vieira LGDR, Araujo T, Manchope LFS, Kuhlhoff MHR, Nagel $\mathrm{V}$, et al. The cost of stroke in a public hospital in Brazil: a oneyear prospective study. Arq Neuropsiquiatr. 2019 Jul;77(6):404-11. https://doi.org/10.1590/0004-282X20190059

10. Vicente VS, Cabral NL, Nagel V, Guesser VV, Safanelli J. Prevalence of obesity among stroke patients in five Brazilian cities: a crosssectional study. Arq Neuropsiquiatr. 2018 Jun;76(6):367-72. https:// doi.org/10.1590/0004-282×20180053

11. Lange MC, Ribas G, Scavasine V, Ducci RD, Mendes DC, Zétola VHF, et al. Stroke recurrence in the different subtypes of ischemic stroke. The importance of the intracranial disease. Arq Neuropsiquiatr. 2018 Oct;76(10):649-53. https://doi.org/10.1590/0004-282X20180095

12. Vieira LGDR, Safanelli J, Araujo T, Schuch HA, Kuhlhoff MHR, Nagel $\mathrm{V}$, et al. The cost of stroke in private hospitals in Brazil: a one-year prospective study. Arq Neuropsiquiatr. 2019 Jul;77(6):393-403. https://doi.org/10.1590/0004-282X20190056

13. Cabral NL, Longo AL, Moro CHC, Amaral CH, Kiss HC. Epidemiologia dos acidentes cerebrovasculares em Joinville, Brasil: estudo institucional. Arq Neuro-Psiquiatr [online]. 1997 Sep;55(3A):357-63. https://doi.org/10.1590/S0004-282X1997000300002
14. da Silva CF, Schwartz J, Belli VDS, Ferreira LE, Cabral NL, França PHC. Ischemic stroke and genetic variants: in search of association with severity and recurrence in a brazilian population. J Stroke Cerebrovasc Dis. 2020 Feb;29(2):104487. https://doi.org/10.1016/j. jstrokecerebrovasdis.2019.104487

15. Cabral NL, Longo A, Moro C, Ferst P, Oliveira FA, Vieira CV, et al. Education level explains differences in stroke incidence among city districts in Joinville, Brazil: a three-year population-based study. Neuroepidemiology. 2011;36(4):258-64. https://doi. org/10.1159/000328865

16. Cabral NL, Franco S, Longo A, Moro C, Buss TA, Collares D, et al. The Brazilian Family Health Program and secondary stroke and myocardial infarction prevention: a 6 -year cohort study. Am J Public Health. 2012 Dec;102(12):e90-5. https://doi.org/10.2105/ AJPH.2012.301024

17. Moro CH, Gonçalves AR, Longo AL, Fonseca PG, Harger R, Gomes DB, et al. Trends of the incidence of ischemic stroke thrombolysis over seven years and one-year outcome: a population-based study in Joinville, Brazil. Cerebrovasc Dis Extra. 2013 Dec;3(1):156-66. https:// doi.org/10.1159/000356984

18. Cabral NL, Muller M, Franco SC, Longo A, Moro C, Nagel V, et al. Threeyear survival and recurrence after first-ever stroke: the Joinville stroke registry. BMC Neurol. 2015 May;15:70. https://doi.org/10.1186/ s12883-015-0317-1

19. Cabral NL, Conforto A, Magalhaes PSC, Longo AL, Moro CHC, Appel $\mathrm{H}$, et al. Intravenous rtPA versus mechanical thrombectomy in acute ischemic stroke: A historical cohort in Joinville, Brazil. eNeurologicalSci. 2016 Apr;5:1-6. https://doi.org/10.1016/j. ensci.2016.04.002

20. Cabral NL, Nagel V, Conforto AB, Magalhaes PS, Venancio VG, Safanelli J, et al. High five-year mortality rates of ischemic stroke subtypes: A prospective cohort study in Brazil. Int J Stroke. 2019 Jul;14(5):491-9. https://doi.org/10.1177/1747493018806197

21. Campos LM, Martins BM, Cabral NL, Franco SC, Pontes-Neto OM, Mazin SC, et al. How many patients become functionally dependent after a stroke? A 3-year population-based study in Joinville, Brazil. PLoS One. 2017 Jan;12(1):e0170204. https://doi.org/10.1371/ journal.pone.0170204

22. Cabral NL, Freire AT, Conforto AB, Dos Santos N, Reis FI, Nagel V, et al. Increase of stroke incidence in young adults in a middleincome country: a 10-year population-based study. Stroke. 2017 Nov;48(11):2925-30. https://doi.org/10.1161/STROKEAHA.117.018531

23. Cabral NL, Cougo-Pinto PT, Magalhaes PS, Longo AL, Moro CH, Amaral CH, et al. Trends of Stroke Incidence from 1995 to 2013 in Joinville, Brazil. Neuroepidemiology. 2016;46(4):273-81. https://doi. org/10.1159/000445060

24. Cabral NL, Nagel V, Conforto AB, Amaral CH, Venancio VG, Safanelli $J$, et al. Five-year survival, disability, and recurrence after first-ever stroke in a middle-income country: A population-based study in Joinvile, Brazil. Int J Stroke. 2018 Oct;13(7):725-33. https://doi. org/10.1177/1747493018763906

25. Ouriques Martins SC, Sacks C, Hacke W, Brainin M, de Assis Figueiredo F, Marques Pontes-Neto O, et al. Priorities to reduce the burden of stroke in Latin American countries. Lancet Neurol. 2019 Jul;18(7):674-83. https://doi.org/10.1016/\$1474-4422(19)30068-7 